\title{
Échanges de coquillages entre amateurs et professionnels
}

L'économie morale des sciences naturalistes

Shell exchanges between amateurs and professionals. The moral economy of naturalist sciences

\section{Elsa Faugère}

\section{OpenEdition}

\section{Journals}

Édition électronique

URL : https://journals.openedition.org/tc/6652

DOI : $10.4000 /$ tc. 6652

ISSN : 1952-420X

Éditeur

Éditions de l'EHESS

\section{Édition imprimée}

Date de publication : 15 décembre 2012

Pagination : 206-223

ISBN : 978-2-7351-1534-

ISSN : 0248-6016

\section{Référence électronique}

Elsa Faugère, «Échanges de coquillages entre amateurs et professionnels », Techniques \& Culture [En ligne], 59 | 2012, mis en ligne le 15 décembre 2015, consulté le 29 septembre 2022. URL : http:// journals.openedition.org/tc/6652; DOI : https://doi.org/10.4000/tc.6652 


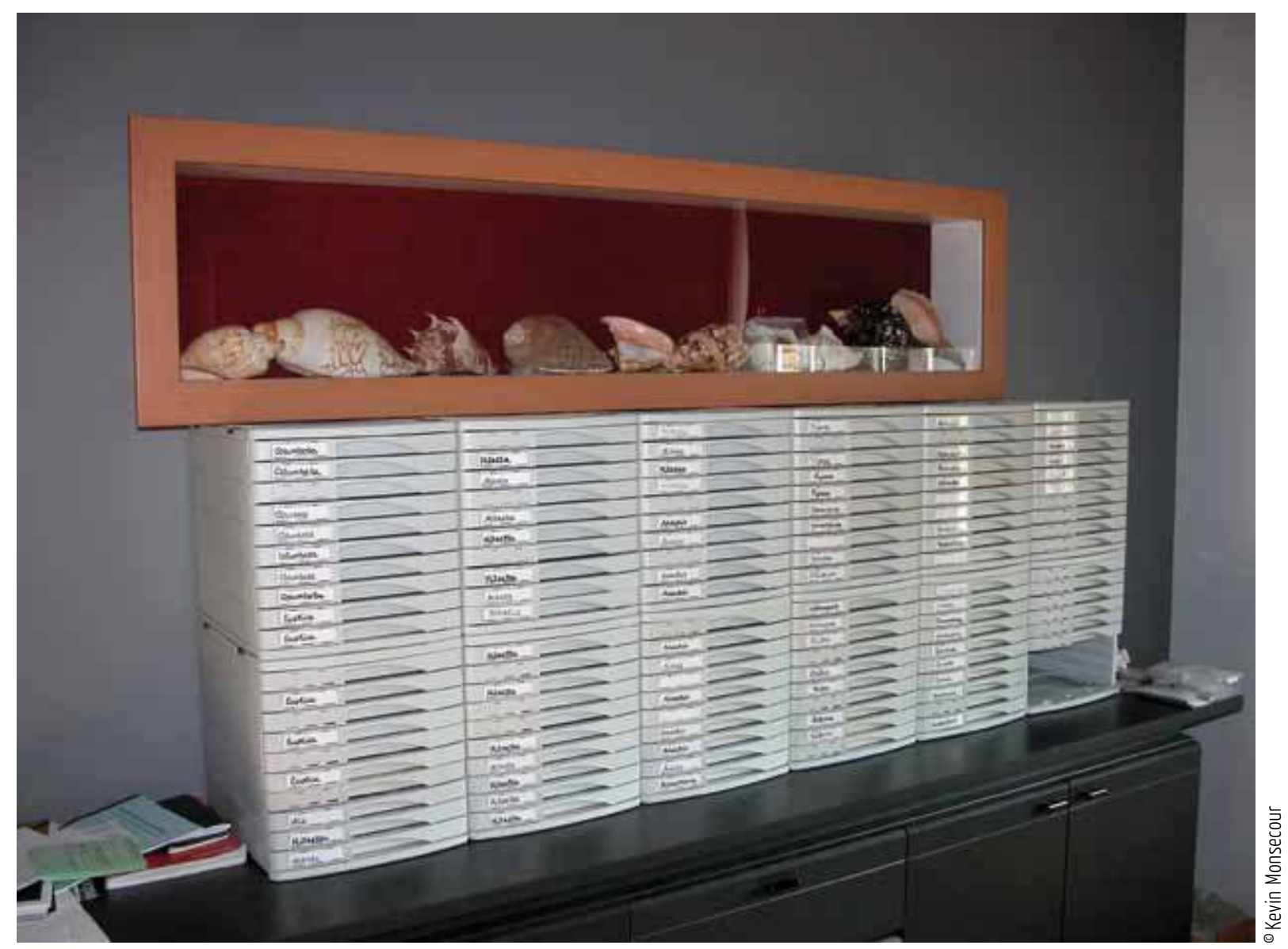




\section{ÉCHANGES DE COQUILLAGES \\ ENTRE AMATEURS ET PROFESSIONNELS}

\section{L'économie morale des sciences naturalistes}

En 2005, j’ai commencé à m'intéresser, en tant qu’anthropologue, aux grandes expéditions naturalistes contemporaines, organisées par le Muséum national d'histoire naturelle de Paris, l'ONG Pro-Natura International et l'Institut de Recherche pour le Développement ${ }^{1}$. J'ai rapidement été attirée et intriguée par deux aspects inattendus dans des recherches scientifiques académiques: la présence de mécènes qui, sous forme de fondations privées, familiales et d'entreprises, financent l'intégralité de ces expéditions (hors salaires des participants); et la présence d'amateurs de toutes sortes (chauffeur de bus, agent immobilier, dentiste, professeurs du secondaire, en activité et en retraite, informaticiens, militaire, chef d'entreprise, médecins, diplomates, etc.) qui, des collectes sur le terrain jusqu'à la description d'espèces nouvelles pour la science constituent une main-d'œuvre bénévole, hétéroclite et bigarrée sans laquelle cette œuvre scientifique de collecte, d'inventaire du vivant et de mise en collection ne pourrait pas se faire.

Ignorant alors l'histoire des sciences naturalistes, je pensais que le mécénat des " grands de ce monde », et le bénévolat « des gens du commun » reflétaient un état très particulier des relations contemporaines entre sciences (naturelles) et société, caractérisée par l'irruption de financements privés dans la recherche académique - sur le modèle nord-américain - (Kohler 1991, 2006; Guilhot 2004; Bory 2008) et le développement de sciences citoyennes dans lesquels les « profanes » co-construisaient les savoirs aux côtés des scientifiques professionnels (Charvolin, Micoud \& Nyhart 2007; Charvolin 2009; Alliage 2011 : 69).

Mais rapidement, au fil de mes lectures et de l'avancement de mes enquêtes ethnographiques j'ai compris que mécénat et bénévolat constituaient en fait deux piliers dans 


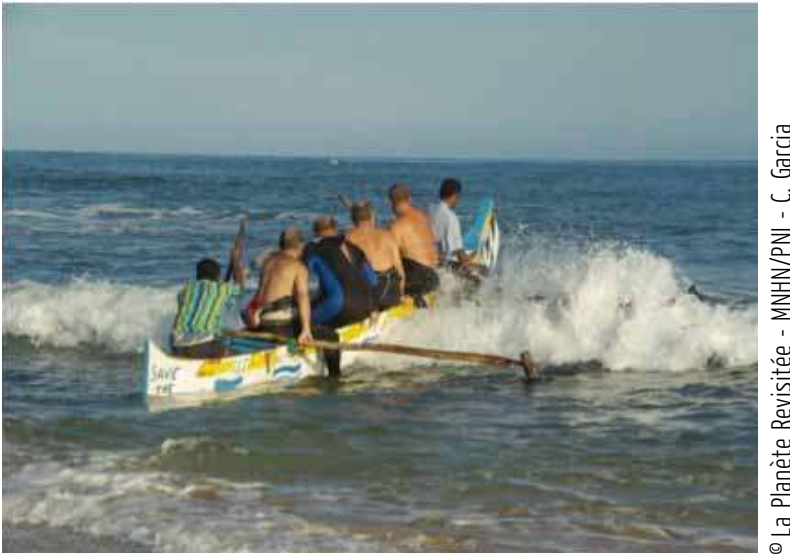

Parmi les nombreux amateurs qui participent aux expéditions naturalistes, il y a toujours des plongeurs qui, en tant que collecteurs des mollusques, jouent un rôle majeur. La réussite d'une expédition repose en partie sur eux, et sur leurs capacités et compétences à repérer, sous l'eau, des habitats susceptibles d'abriter des mollusques. Dans ces expéditions, ils utilisent trois grandes techniques de collecte: le brossage, le suçage et la collecte à vue. Sur cette photo prise en juin 2010 lors de l'expédition Atimo Vatae à Lavanono (Madagascar), on voit notamment Jacques Pelorce, amateur et collectionneur des coquillages de Méditerranée, et Patrice PetitDevoize, amateur de coquillages, responsable de la Commission Biologie Environnement de la Fédération Française de Plongée, partir en mer pour effectuer leurs collectes quotidiennes.

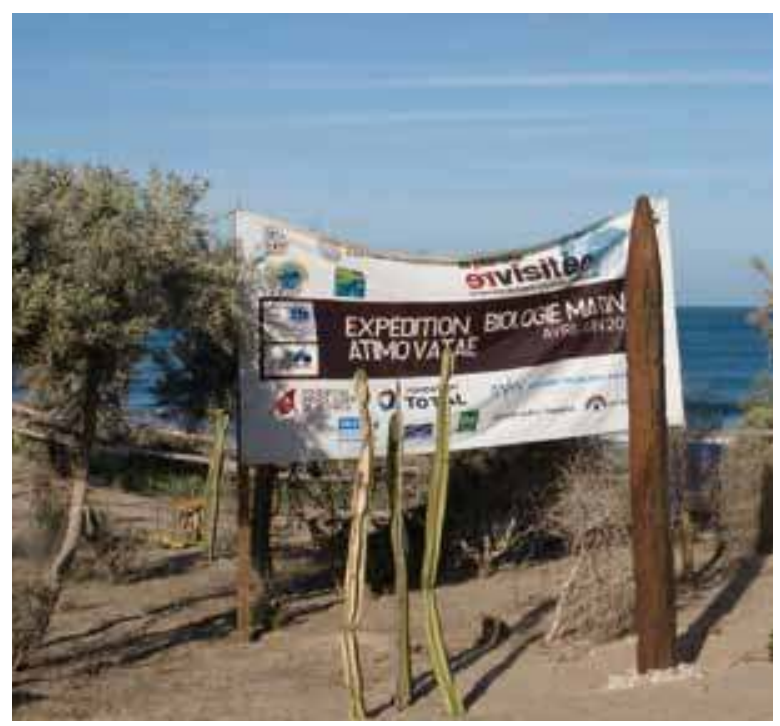

๑ La Planète Revisitée - MNHN/PNI - A. Fedosov pour la soif $»)^{3}$. l'histoire des sciences naturalistes qui, au moins depuis le XIXe siècle, s'appuie sur la participation de mécènes, de bénévoles et d'amateurs (Kohler 1991, 2006) (voir Guichard dans ce numéro). La pérennité du mécénat et du bénévolat dans l'histoire des sciences naturalistes et dans la construction des savoirs scientifiques sur la nature m'ont conduite à faire l'hypothèse de l'existence d'une économie morale spécifique à ces sciences naturalistes de terrain ${ }^{2}$ (Daston 1995; Fassin 2009; Strasser 2011, 2012).

L'une des composantes de cette économie morale réside dans des valeurs, des passions et des centres d'intérêt communs aux amateurs et aux professionnels, au premier rang desquels figurent les points de vue suivants: les savoirs scientifiques sur la biodiversité sont supérieurs aux autres types de savoirs; l'entreprise d'inventaire et de collecte du vivant est de la plus haute importance en cette période de $\sigma^{e}$ crise d'extinction des espèces; et enfin, tous sont, ou ont été, des collectionneurs (de papillons, de coquillages, de sachets de baguettes, de boîtes de sardines, de stock de légumes, de fossiles, de billets de banque, etc.).

Mais, au-delà du partage de ces valeurs et de ces centres d'intérêt, mes enquêtes ethnographiques montrent que ce qui fait tenir ce collectif composite et éclectique, depuis parfois plus de trente ans, ce sont des échanges de toutes sortes, et plus particulièrement des échanges de coquillages, des dons et des contre-dons qui circulent selon des règles bien codifiées, même si certaines ne sont énoncées qu'à voix basse (comme « la poire

L’objet de cet article, dans le cadre de ce Thema consacré aux itinéraires de coquillages, est ainsi de montrer que l'une des dimensions - peut-être même la principale - de cette économie morale réside dans une double circulation de coquillages qui des amateurs vers les professionnels consiste en des dons d'holotypes ${ }^{4}$, et en sens inverse, des professionnels vers les amateurs en des dons de paratypes.

Dans une première partie, nous suivrons Luigi, un marchand italien de coquillages qui s'est spécialisé depuis 1999 dans l'achat et la revente de coquillages du Sud de Madagascar. Nous verrons alors comment en tant que découvreur et descripteur de dizaines d'espèces nouvelles pour la science, selon l'expression consacrée, Luigi a tissé des liens étroits avec les malacologues professionnels du Muséum national d'histoire naturelle de Paris.

Dans une seconde partie nous suivrons Kevin et Koen, deux amateurs belges de « haut niveau » à qui, en échange et en remerciement du long travail de tri à la famille et à l'espèce

On aperçoit sur cette photo prise à Lavanono la banderole où figure l'ensemble des mécènes de l'expédition Atimo Vatae. Elle est restée affichée de mai à juin 2010 pendant toute la durée de l'expédition marine à Fort Dauphin (Madagascar) d'abord puis à Lavanono. Les trois plus importants mécènes étaient la Fondation Prince Albert II de Monaco, la Fondation d'entreprise Total et la fondation familiale Stavros Niarchos. 
qu'ils acceptent de faire sur les collectes des campagnes du Muséum, les malacologues professionnels leur donnent les paratypes pour leur collection personnelle. Considérés par certains de ces amateurs comme un paiement, en raison de la valeur marchande de certaines espèces, les coquillages retrouvent là, d'une certaine façon, leur ancienne fonction de monnaie. Mais nous verrons que ce point de vue n'est pas partagé par tous.

Au-delà des savoirs scientifiques sur la nature, ce que ces circuits d'échanges fabriquent, c'est du lien social. L'appartenance à un collectif, à une «famille », m'ont même dit certains amateurs-collectionneurs, compte beaucoup à leurs yeux. C'est souvent ainsi qu'ils expliquent les raisons pour lesquelles ils viennent chaque année, parfois de très loin, à la Bourse aux coquillages de Paris, " pour retrouver les amis et les copains », et les raisons pour lesquelles ils acceptent de passer parfois beaucoup de temps, bénévolement, à étudier le matériel des malacologues du Muséum de Paris, appelé par certains professionnels, « la maison».

\section{Luigi, le marchand italien, donne ses holotypes au Muséum}

Luigi, âgé d'une soixantaine d'années, est un informaticien italien à la retraite qui se passionne pour les coquillages, les achète, les vend et les collectionne, depuis plus de 30 ans. Spécialiste au départ de la faune de Somalie, les conflits politiques qui, dans les années 1990, ont frappé ce pays l'ont poussé à changer de destination. Dès 1999, il se focalise et se spécialise dans les coquillages du Sud de Madagascar où il vient plusieurs fois par an. Son mode opératoire est simple: il achète les coquillages aux pêcheurs, aux femmes malgaches, et sur les marchés locaux et notamment celui de Tuléar qui est particulièrement réputé. Il a su fidéliser un réseau de collecteurs qui lui vendent prioritairement leurs collectes, même s'il n'est pas le seul marchand collectionneur italien (voir dans ce numéro: Christine Demmer). À chaque voyage il repart avec deux valises et cinquante kilogrammes de coquillages.

Et il les vend notamment à la bourse de Paris où chaque année il tient son stand de coquillages du Sud de Madagascar. Considérée comme la plus importante bourse aux coquillages du monde, la bourse de Paris rassemble le premier week-end du mois de mars, tous les marchands de coquillages, petits et grands, amateurs et professionnels (qui sont une

Luigi et Sonia, la conservatrice du Muséum d'histoire naturelle de Saint-Denis de La Réunion, à Fort Dauphin, lors de l'expédition Atimo Vatae, au stand d'un marchand malgache de coquillages. Guidée et conseillée par Luigi sur les prix et la valeur de chacun des coquillages, Sonia achète des spécimens pour une exposition qu'elle prépare au Muséum de Saint-Denis, mai 2010.

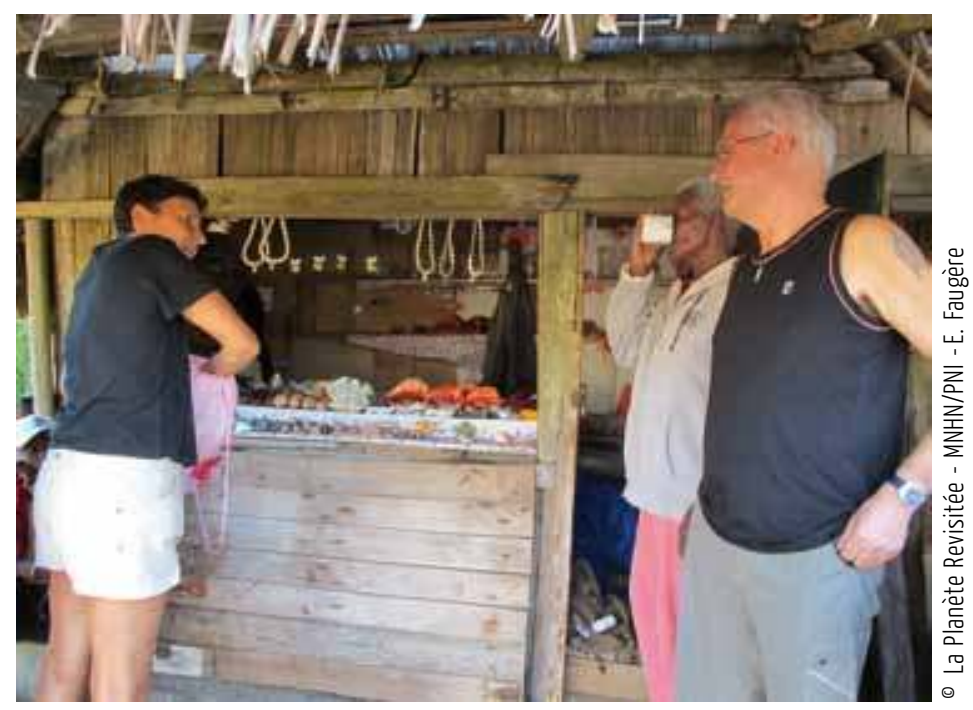




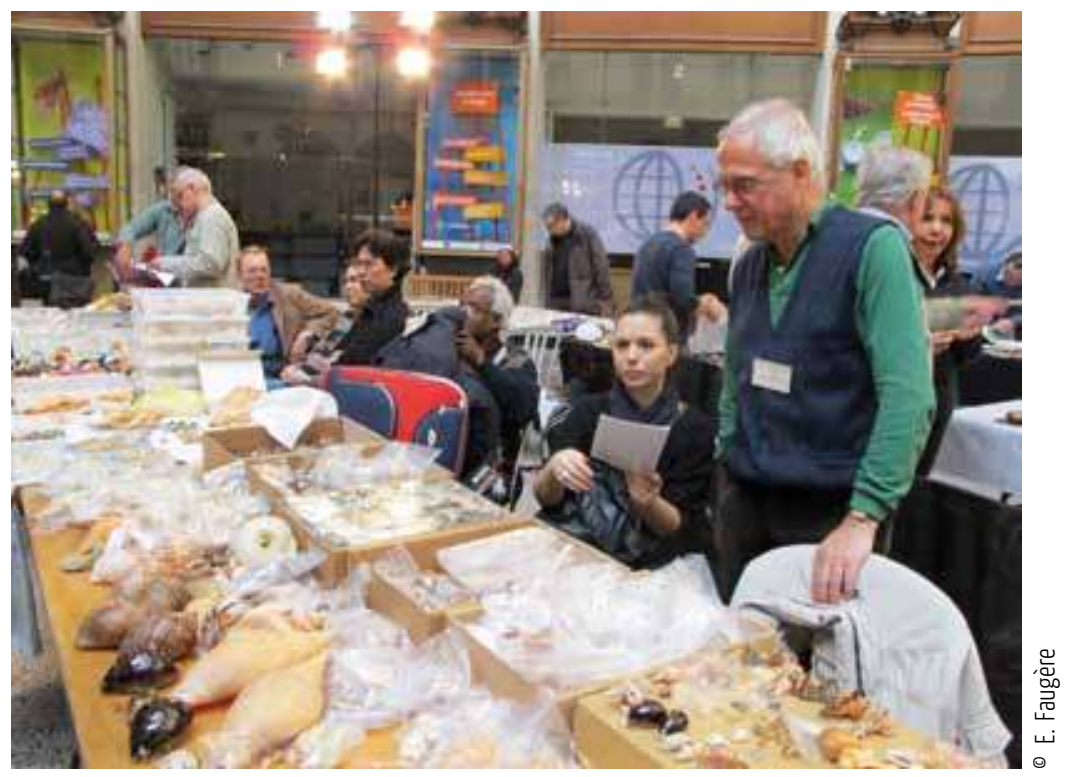

petite poignée), des collectionneurs de toutes sortes, ainsi que certains scientifiques.

C'est là que Luigi a rencontré Philippe B., professeur de malacologie au Muséum national d'histoire naturelle de Paris, il y a une vingtaine d'années.

Considéré comme l'un des plus grands malacologues contemporains, Philippe, avec son équipe de fidèles collaborateurs au premier rang desquels Virginie, Philippe M., et Pierre, organisent depuis les années 1980 des campagnes de collecte et d'exploration marines principalement dans le Pacifique Sud ${ }^{5}$ (Bouchet, Héros, Lozouet, Maestrati 2008) et, depuis les années 2000, de grandes expéditions d'inventaires de la biodiversité, déjà évoquées.

Personnage charismatique, véritable entrepreneur de la science, excellent

Luigi, derrière son stand de coquillages à la bourse de Paris, en mars 2011

Luigi lors de l'expédition Atimo Vatae à Fort Dauphin, sud de Madagascar, mai 2010. II montre fièrement le Lambis Christine qu'il s'est fait tatouer sur le bras, nouvelle espèce très rare et très chère quiil a découverte à Tuléar en 2000. II raconte: « le Lambis c'est le coquillage avec les épines comme ça. J'en ai trouvé un nouveau et j’ai donné le nom Lambis Christine. Et actuellement, il n'y a que cinq spécimens trouvés en 12 ans. C'est très rare. Alors, pour la publicité je me la suis fait tatouer (il me montre son tatouage) sur son bras! Quand je vais à la bourse aux coquillages, je leur montre: tu vois le coquillage comme ça (rires)! Le prix de la Lambis Christine c'est 3000 ou 3500 euros. Mais c'est une exception parce que la plupart des coquillages même les rares, ils valent entre 100 et 500 euros et les coquillages communs c'est 5 euros, 10 euros, 15 euros. »

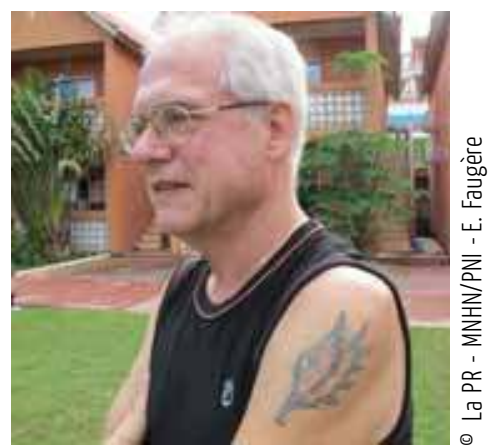

communicant, Philippe incarne une figure atypique du scientifique. Il n'hésite pas à côtoyer des univers très éclectiques et non académiques comme la tour Total à la Défense où il donne régulièrement des conférences devant un parterre d'actionnaires endormis ${ }^{6}$, les bourses aux coquillages et notamment celle de Paris où il a su tisser des liens de travail, de confiance et d'amitié avec de nombreux amateurs-collectionneurs-marchands de coquillages, français, belges, néerlandais, italiens, américains, espagnols, philippins, brésiliens, etc.

À l'occasion de cette bourse des coquillages, Philippe organise généralement une visite de la zoothèque, de la typothèque - la collection des types -, de la bibliothèque et du laboratoire de malacologie du Muséum. Au cours d'un entretien en février 2012, il se souvient de Luigi qui avait participé à l'une de ces visites au début des années 1990. Il raconte:

«J'avais accueilli un certain nombre de gens comme Luigi au Muséum et je leur avais fait visiter la zoothèque, la bibliothèque, etc. Je me rappelle Luigi en particulier, il était honoré et impressionné! Et il avait fait un article dans un trimestriel italien qui s'appelait La Conchiglia qui a cessé de paraître mais qui avait beaucoup d'impact dans le milieu des amateurs et collectionneurs. Et dans son article en gros il disait qu'il n'y a rien de mieux que le Muséum de Paris! Et à partir de ce moment-là, il a commencé à déposer les holotypes de ses espèces nouvelles ici, y compris ceux pour lesquels je n'étais pour rien! Il allait en voyage à Zanzibar, il revenait avec des espèces nouvelles, il les décrivait, et il déposait le type ici voilà ».

L'holotype, également appelé type est le spécimen à partir duquel l'espèce a été décrite. Tous les types de mollusques du Muséum de Paris sont conservés dans des salles particulières appelées la typothèque, qui sont placées sous la responsabilité de Virginie. Pour les malacologues, amateurs et professionnels, la typothèque est un véritable «sanctuaire » de coquillages puisqu'il contient l'ensemble des types des espèces découvertes et décrites à ce jour:

«Au Muséum, Virginie Héros est la grande prêtresse qui garde le sanctuaire de notre typothèque: tout type n'y entre qu'après qu'elle a vérifié son statut au regard de la 
description originale et du Code de Nomenclature; tout type n'en sort qu'avec son accord, assorti d'un «fantôme » ou d'une feuille de prêt ». (Bouchet, Mermet 2007 : 97-98)

Le prestige d'un muséum d'Histoire naturelle repose sur le nombre de types qu'il possède. Le Muséum de Paris, avec ses 12000 types de mollusques arrive ainsi en $3^{\mathrm{e}}$ rang mondial, loin derrière le Natural History Museum de Londres (qui possède 27000 types) et à égalité avec le National Museum of Natural History de Washington (Bouchet, Mermet 2007 : 97). L'obtention et le dépôt des types sont donc des activités tout à fait essentielles dans la vie d'un muséum d'Histoire naturelle comme celui de Paris.

Pourquoi les amateurs marchands de coquillages comme Luigi préferent-ils déposer les types au Muséum plutôt que de les vendre à la bourse aux coquillages? D’après les malacologues professionnels et amateurs que j'ai rencontrés, donner ses types à un musée constitue une tradition de la malacologie. «Cela ne se fait pas de vendre les holotypes, alors que de telles pratiques existent en entomologie. Personne ne brise cette loi non écrite qui est un code de bonne conduite dans le milieu de la malacologie », selon un malacologue professionnel.

L'examen minutieux du chemin parcouru par les coquillages de Luigi éclaire ces pratiques.

Dans un entretien fait à Madagascar en mai 2010, Luigi raconte que depuis son premier voyage dans ce pays en 1999, il a décrit 120 nouvelles espèces de mollusques achetées à des pêcheurs, à des femmes malgaches et sur les marchés locaux. Quand il collecte des spécimens qu'il n'a jamais vus auparavant et qu'il considère comme étant peut-être des espèces nouvelles, il s'empresse de les décrire dans une revue italienne de malacologie qui s'appelle Mostra Mondiale Malacologia. Dirigée par deux frères, amateurs, marchands et collectionneurs italiens, qui ont créé un musée des coquillages à Cupra Maritima sur la côte Adriatique en Italie, le simple fait de publier une description d'une espèce nouvelle de coquillage, même dans une petite revue amateur de malacologie, donne immédiatement une valeur marchande à cette espèce nouvelle. Ainsi, Luigi, ou tout autre amateur et marchand de coquillage qui vient de décrire une nouvelle espèce (ou ce qu'il croit être une nouvelle espèce) peut dès lors vendre les autres spécimens de cette espèce.

Déposer les types au Muséum donne de la crédibilité et de la légitimité scientifiques à l'espèce nouvellement décrite, ainsi qu'à son descripteur, et permet en l'occurrence à Luigi d'entretenir de bonnes relations avec l'équipe de malacologie du Muséum.

«Quand tu as ton matériel, tu peux avoir 1, 2, 3, 10, 100 spécimens et le spécimen sur lequel tu fais le travail de description alors tu dois le désigner comme holotype. C'est le spécimen qui représente l'espèce. Les holotypes tu es moralement obligé de les donner au Muséum, parce que c'est la pièce de référence. Quand tous les spécialistes dans le monde cherchent une information sur cette espèce, ils savent que les holotypes sont au Muséum de Paris. Les autres spécimens que tu as utilisés pour préparer le travail s'appellent les paratypes. Et les paratypes peuvent rester dans les collections de l'auteur ou dans les collections des muséums. J'ai une petite mais très belle collection de paratypes des coquillages que j'ai décrits, j'ai 500 ou 600 spécimens » (entretien avec Luigi, mai 2010, Fort Dauphin, Madagascar).

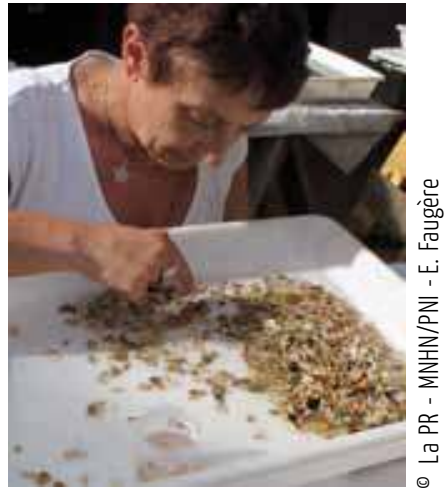

Virginie Héros en train de trier la laisse de mer, laboratoire de plein air de Fort Dauphin et de Lavanono, expédition Atimo Vatae, Madagascar, juin 2010.

Des amateurs italiens, Ricardo et Francesco, avec un couple d'amis, à la typothèque du Muséum, en mars 2012.

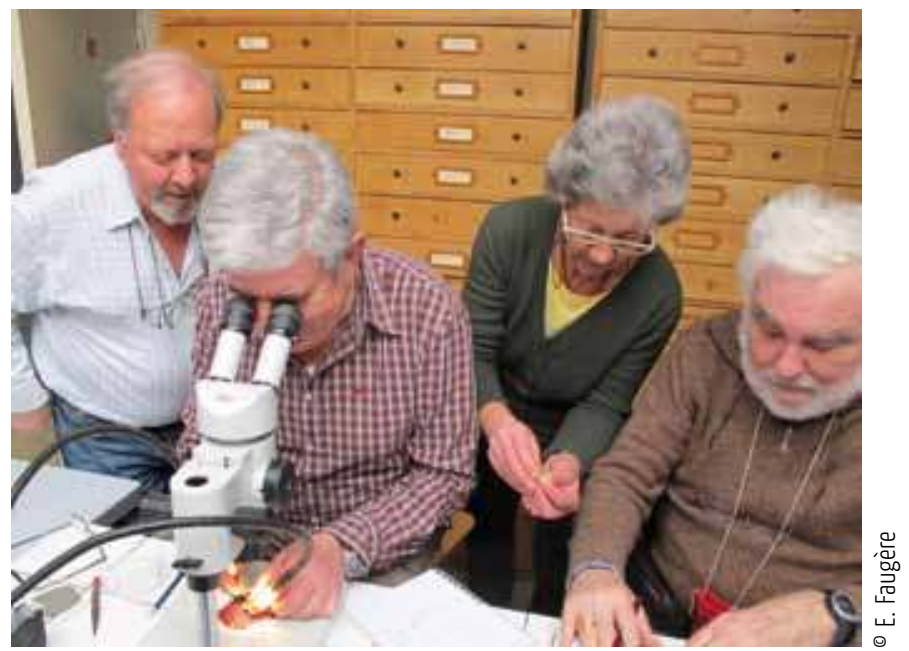


Même si les malacologues professionnels considèrent, parfois, que les descriptions d'espèces nouvelles publiées dans certaines revues d'amateurs sont mauvaises (notamment parce qu'ils n'ont pas fait le long et laborieux travail bibliographique qu'ils auraient dû faire), il est important pour eux d'entretenir de bonnes relations avec ces amateurs qui donnent leurs types au Muséum et qui, ainsi, participent à leur échelle et à leur manière à cette entreprise d'exploration, d'inventaire et d'archivage du vivant.

Luigi Bozzetti, Philippe Bouchet, Laure Corbari, Sarah Amiraly autour d'un bac contenant les spécimens fraîchement pêchés, laboratoire de plein air de Fort Dauphin, expédition Atimo Vatae, Madagascar, mai 2010.

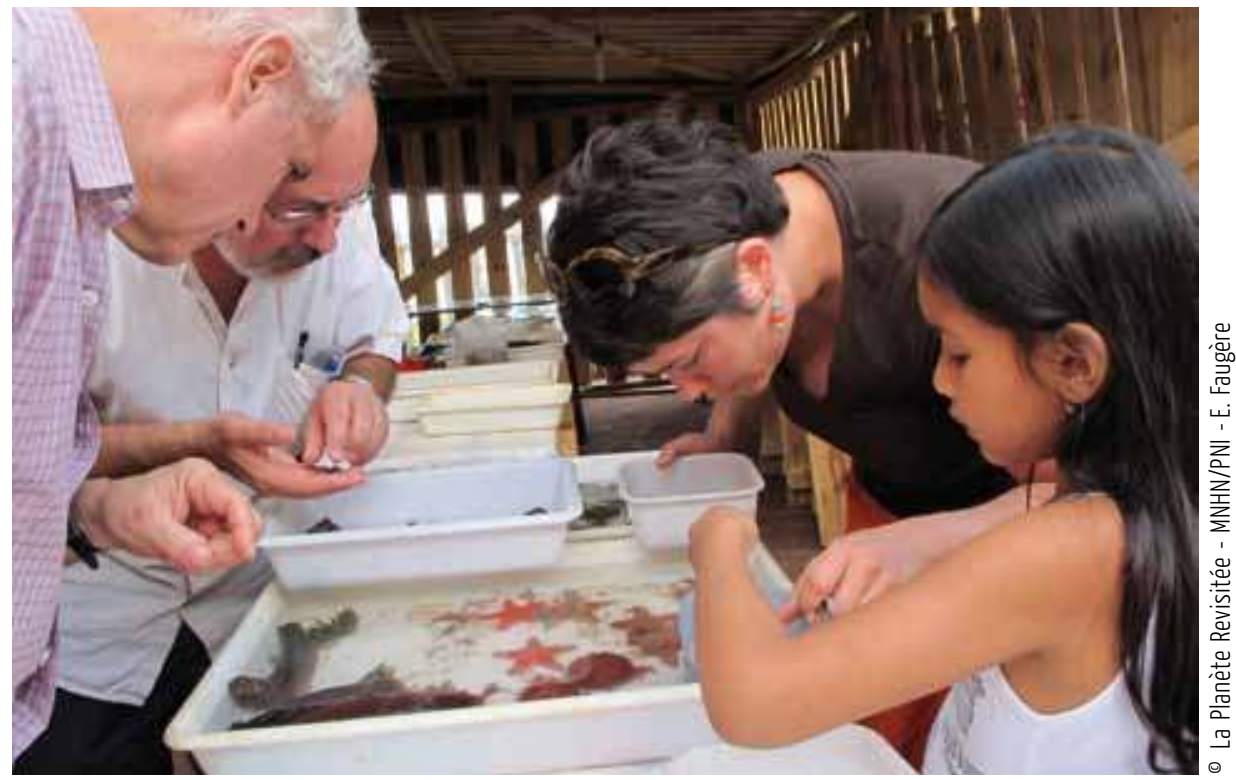

En remerciement des dons de types que Luigi a fait au Muséum de Paris, plutôt qu'à un autre musée, et de l'important travail de découverte d'espèces nouvelles du Sud malgache qu'il a accompli, et à partir duquel l'expédition Atimo Vatae a été planifiée et organisée, Philippe et son équipe lui ont fait un contre-don en l'invitant à participer à cette expédition en 2010: «Donc je dirai qu'amener Luigi à Madagascar c'était aussi une façon de le remercier et de lui renvoyer l'ascenseur » (Philippe, février 2012). De nombreux autres contre-dons de toutes sortes existent. Par exemple Virginie, chargée de la typothèque est toujours très disponible et réactive aux demandes diverses et variées émanant de leur cercle d'amateurs, et leur rend de nombreux services qui permettent de consolider les liens.

Sans entrer ici dans le détail de ces micro-échanges qui risqueraient de nous éloigner de la circulation des coquillages proprement dite, je voudrais juste évoquer un autre type d'échanges de coquillages que les malacologues professionnels appellent « la poire pour la soif $»$. Lorsque, au cours d'une campagne en mer, un dragage remonte de nombreux spécimens d'une même espèce de mollusque qui possède une forte valeur marchande, les malacologues professionnels mettent de côté plusieurs de ces spécimens. Ils savent en effet qu'ils pourront servir ultérieurement de monnaie d'échange pour inciter un marchand ou un amateur collectionneur à déposer ses types au Muséum de Paris plutôt que dans un autre muséum, ou pour les dédommager de l'avoir fait. De manière amusante 
et anecdotique, les coquillages renouent ici avec leur ancienne fonction de monnaie (voir dans ce numéro: Francis Dupuy).

L'expression même de « monnaie de coquillages » est d'ailleurs utilisée, dans certains types d'échanges par certains amateurs appartenant à une deuxième figure dont il va maintenant être question.

\section{Les paratypes comme monnaie de coquillages}

\section{les cas de Kevin et de Koen}

Cette seconde figure d'amateurs collectionneurs de coquillages se distingue nettement du premier type incarné par Luigi. Ils sont majoritairement Belges et Hollandais, pays dans lesquels existe une vive tradition de malacologie amateur de haut niveau, pour des raisons qu'il serait bien trop long d'expliquer ici. Dans ce long itinéraire qui mène les mollusques du fond des mers aux étagères du Muséum de Paris (voir dans ce numéro: David Dumoulin), Luigi se situe en début de chaîne, au moment de la collecte. Même s'il ne collecte pas lui-même, il va sur le terrain acheter aux pêcheurs malgaches. Kevin et Koen, quant à eux, se situent à l'autre extrémité de la chaîne, celle qui, en fin de parcours, réceptionne les spécimens déjà triés famille par famille. Ils sont chargés, par Philippe et son équipe, d'aller plus loin dans le tri et de trier certaines familles à l'espèce.

Depuis l'âge de deux ans, Koen, fils d'un artiste peintre qui lui a « montré la beauté dans la nature, les arbres et les coquillages » collectionne les coquillages. Aujourd'hui âgé d'une quarantaine d'années, ce qui est plutôt jeune dans l'univers masculin et âgé des amateurs collectionneurs et marchands de coquillages, il est chauffeur de bus à mi-temps. Cela, dit-il, lui laisse le temps de lire des livres de malacologie et de corriger ses manuscrits d'articles dans les embouteillages.

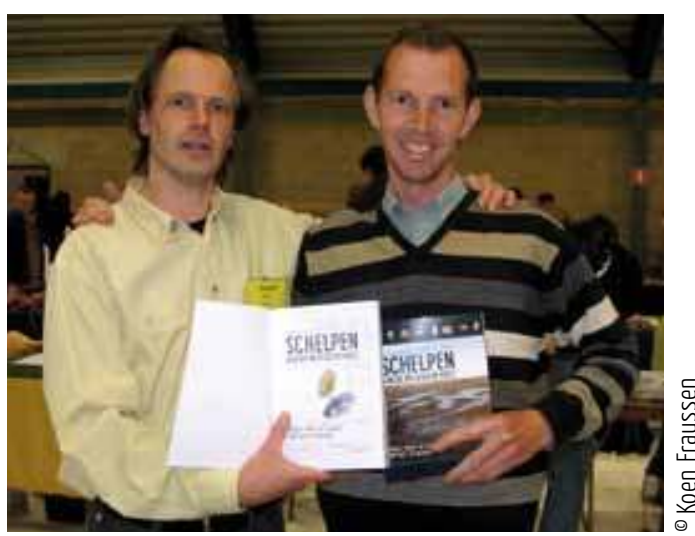

\section{En bas à gauche :}

Koen Fraussen et son co-auteur Stefaan Wera, biologiste, cofondateur et directeur de la logistique d'Okapi Sciences. Ils montrent fièrement leur livre sur les coquillages de Belgique, "Schelpen aan de Belgische Kust », à la bourse aux coquillages d’Anvers, organisée par la société belge de conchyliologie « Gloria Maris », septembre 2010.

\section{Ci-dessous :}

Koen Fraussen avec les pêcheurs de crevettes dans la Mer du Nord, parti pêcher avec eux, à 8 mètres de profondeur, des coquillages pour sa collection personnelle, juillet 2002.

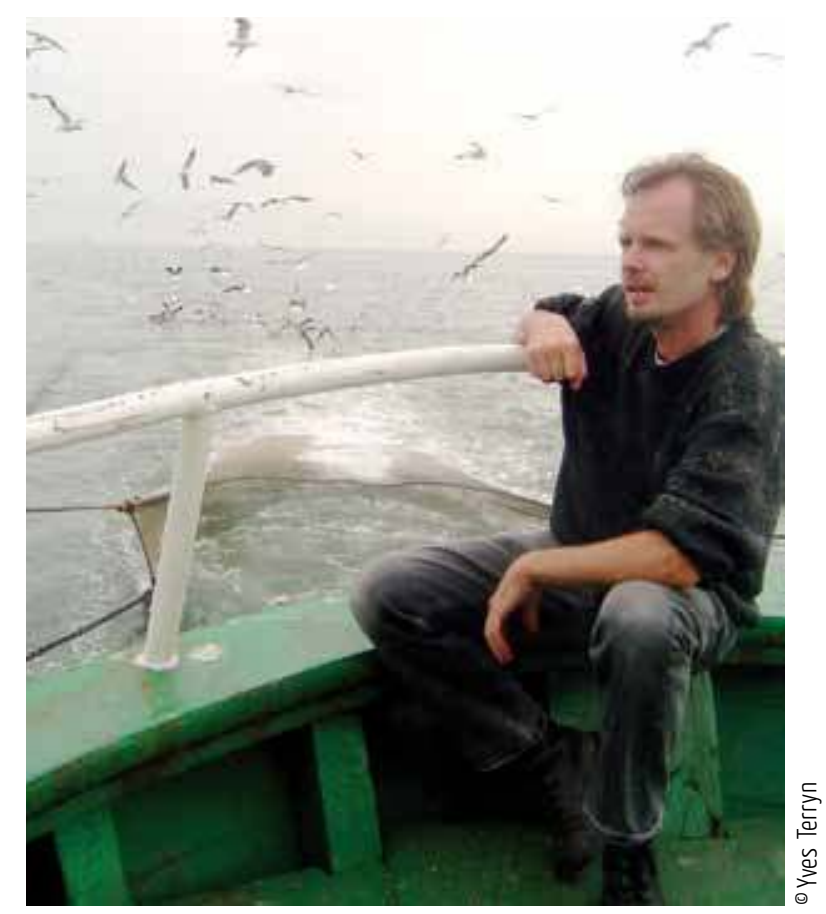




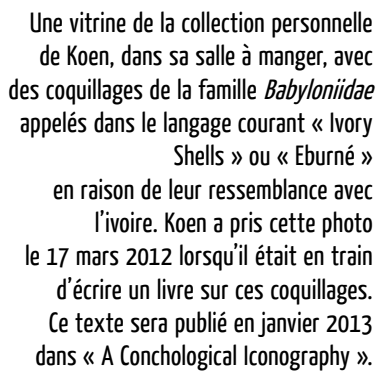

Une vitrine de la collection personnelle de Koen, dans sa salle à manger, avec appelés dans le langage courant « Ivory Shells » ou « Eburné » aison de leur ressemblance avec mars 2012 lorsqu'il était en train d'écrire un livre sur ces coquillages. dans « A Conchological Iconography ».

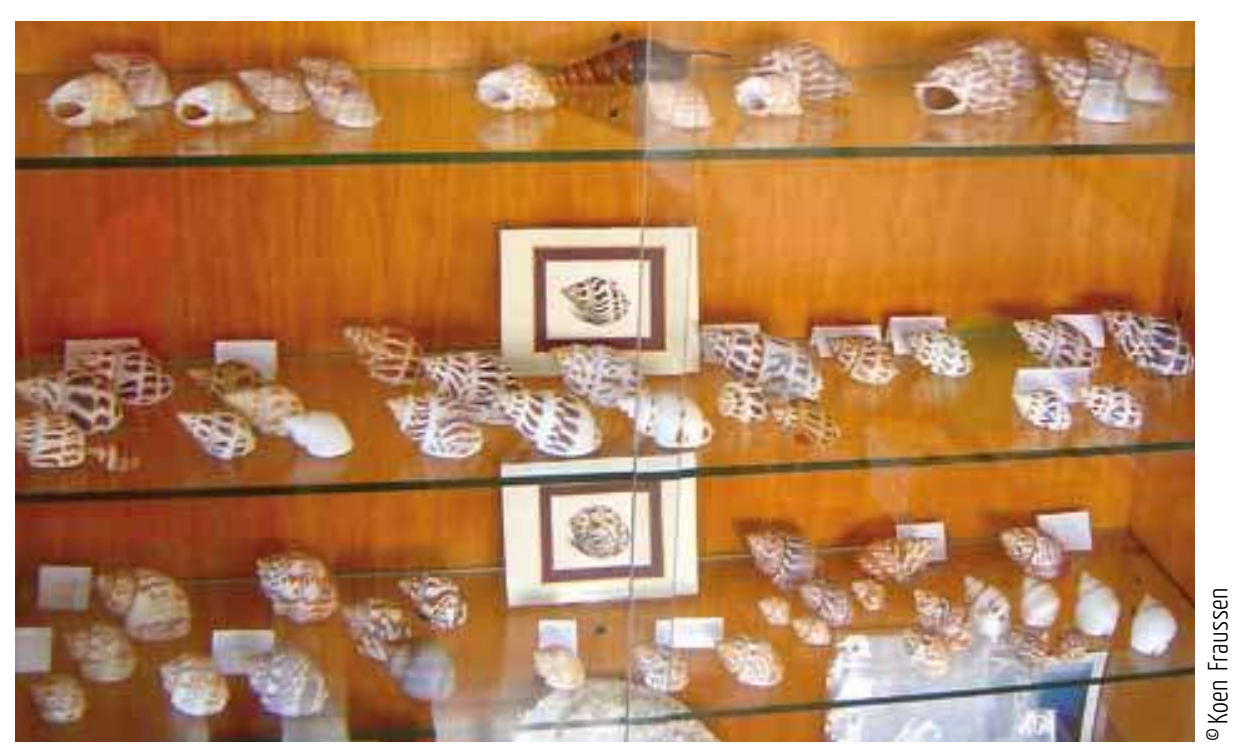

Koen fait en effet partie de la catégorie des amateurs de « haut niveau », comme les qualifient les malacologues professionnels. Dans la subtile échelle qui permet de distinguer et de positionner les amateurs-collectionneurs les uns par rapport aux autres, le principal critère pour appartenir à la plus haute caste - celle des amateurs de haut niveau - est de publier dans des revues considérées, par les malacologues professionnels et amateurs, comme de bonne tenue scientifique, même si ce ne sont que des revues d'amateurs. Koen fait partie de ces gens-là.

Il a rencontré Philippe et son équipe du Muséum en 1995 par l'intermédiaire de Guido, l'un des plus grands marchands de coquillages qui a bâti aux Philippines une véritable entreprise de commerce des coquillages, et qui est également l'un des grands éditeurs de livre scientifique de malacologie.

Étant spécialiste d'un groupe qui s'appelle les Buccinidae, il s'est vu confier la responsabilité d'étudier tous les buccins des campagnes océanographiques du Muséum de Paris, ce qu'il fait depuis lors.

En échange de ce travail de tri et d'identification des espèces de buccins, Philippe lui donne un paratype. Koen explique:

« Le travail scientifique et le commerce ça ne s’accorde pas bien. Au Muséum il y a des spécimens qui viennent de 1000 mètres de profondeur et je dois les nommer. En échange, Philippe me donne un paratype. Parfois, il y a 200 spécimens d'une même espèce dans un peu d'alcool et quand je viens ici à la bourse aux coquillages, je vois ces spécimens en vente à 40 euros. C'est bizarre, ça fait des interférences. [...] Philippe me donne un paratype mais ce n'est pas comme un paiement parce que parfois le paratype il ne vaut rien tellement il est moche! Il existe des coquilles d'eau profonde qui ne ressemblent à rien et qui sont abîmées. Ce n'est donc pas un paiement, c'est juste pour que j'aie aussi un exemplaire dans ma collection personnelle. Lorsque je travaille sur le matériel du Muséum, ça me permet de faire de la comparaison. Si jamais je ne gardais aucun spécimen dans ma collection personnelle, je ne pourrais pas comparer les coquillages que je reçois pour les étudier. C'est donc mieux d'avoir chez soi un spécimen à regarder au microscope pour comparer et être certain de ne pas se tromper » (Entretien avec Koen, bourse aux coquillages de Paris, mars 2012). 
Le point de vue de Koen est intéressant. Alors qu'il sait que parfois, certains spécimens ont une valeur marchande (dans l'extrait d'entretien ci-dessus il évoque le cas de certains qui valent 40 euros pièce), le don de paratype que Philippe lui fait en échange du travail de tri et d'identification qu'il accomplit sur le matériel du Muséum n'est pour lui en aucun cas un paiement en monnaie de coquillages. À ses yeux les spécimens de coquillages qu'il a dans sa collection personnelle jouent un tout autre rôle qui est un rôle d'étude et de travail scientifiques. Pris dans un réseau d'échanges avec les malacologues du Muséum dont l'objectif est l'étude scientifique de certaines familles de mollusques collectées lors de campagnes organisées par le Muséum, Koen considère les dons de paratypes comme venant améliorer le potentiel descriptif et scientifique de sa collection personnelle. Le travail de frontière entre univers marchand et non marchand est souvent subtil et toujours à l'œuvre dans les entretiens que j'ai fait auprès des malacologues amateurs et professionnels. Leur objet d'étude étant parfois objet de science et de commerce, ils doivent souvent rappeler la frontière et la distinction entre le « coquillage monnaie » et le « coquillage savoir».

Mais, d'autres amateurs ont un autre point de vue tout à fait intéressant aussi. C'est le cas d'un très proche ami de Koen, Kevin qui réside à 300 mètres de chez lui en Belgique. Professeur de sciences naturelles au collège, et de dix ans son cadet, Kevin est, avec son frère David, un autre amateur-collectionneur de haut niveau. La manière dont il en est venu à s'intéresser aux coquillages est typique. Il raconte:

« C'est d'abord mon frère qui a commencé à s'intéresser aux coquillages. Mais c'est une histoire très intéressante. Koen habite à Aarschot et mon oncle était un copain de Koen à l'école. Du coup, mon oncle a commencé les coquillages parce que Koen en faisait. Vers 20 ans, mon oncle a arrêté. Mais il avait poussé mon frère qui, à 12 ans, ramassait systématiquement des coquillages quand on partait en vacances à la mer, en France ou en Angleterre. Et moi, je l'ai toujours aidé à chercher les coquillages, mais je n'avais pas de collection à moi. Ce n'est que vers 15 ou 16 ans que j'ai commencé à collectionner quelques groupes ». (Entretien avec Kevin, bourse aux coquillages de Paris, mars 2012).

L'histoire de Kevin est assez exemplaire de celle de nombreux naturalistes, amateurs et professionnels.

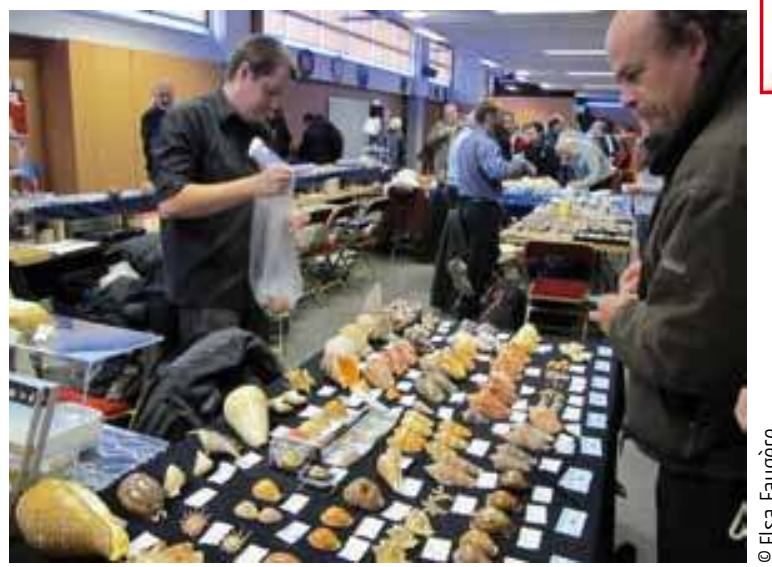

De droite à gauche :

Kevin Monsecour, en train de disposer et d'arranger ses coquillages de la famille Angariidae lors d'une exposition intitulée « Het is maar een stukje kalk! » (Ce n'est que du calcaire!) organisée au musée Stedelijk Museum Aarschot en Belgique par la société savante belge «Gloria Maris » pour fêter son 50 anniversaire (cf. affiche de l'exposition ci-dessous).

Kevin, derrière son stand à la bourse de Paris, mars 2012.

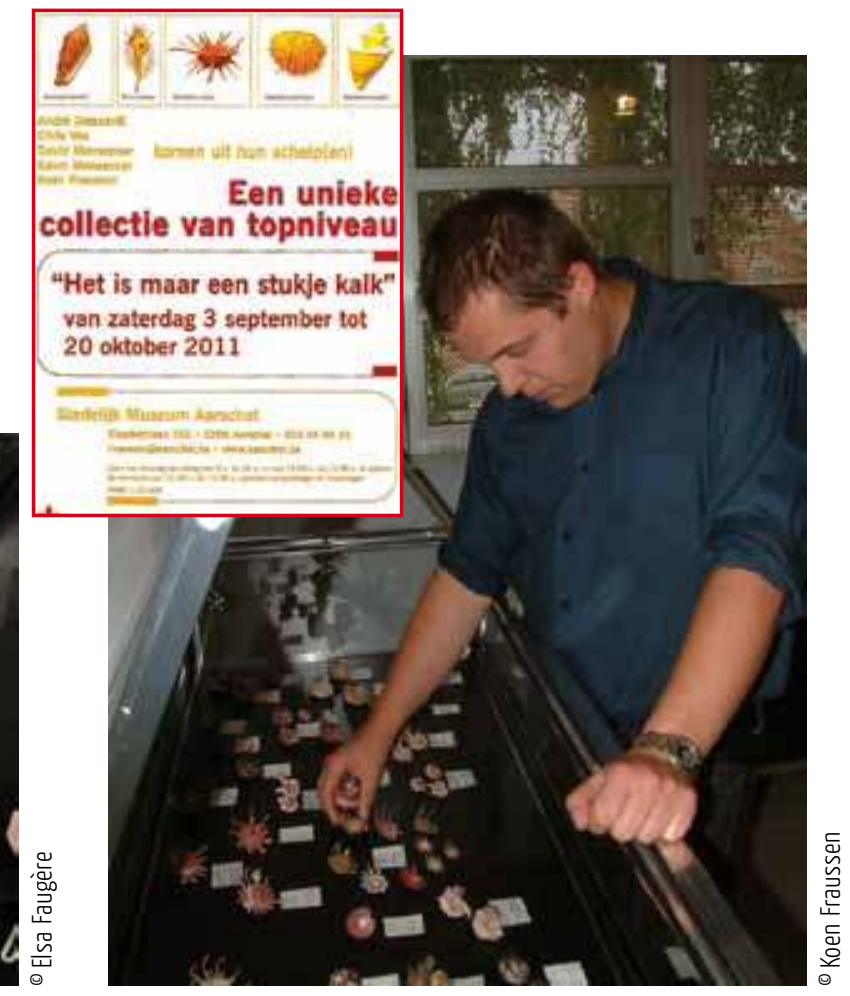




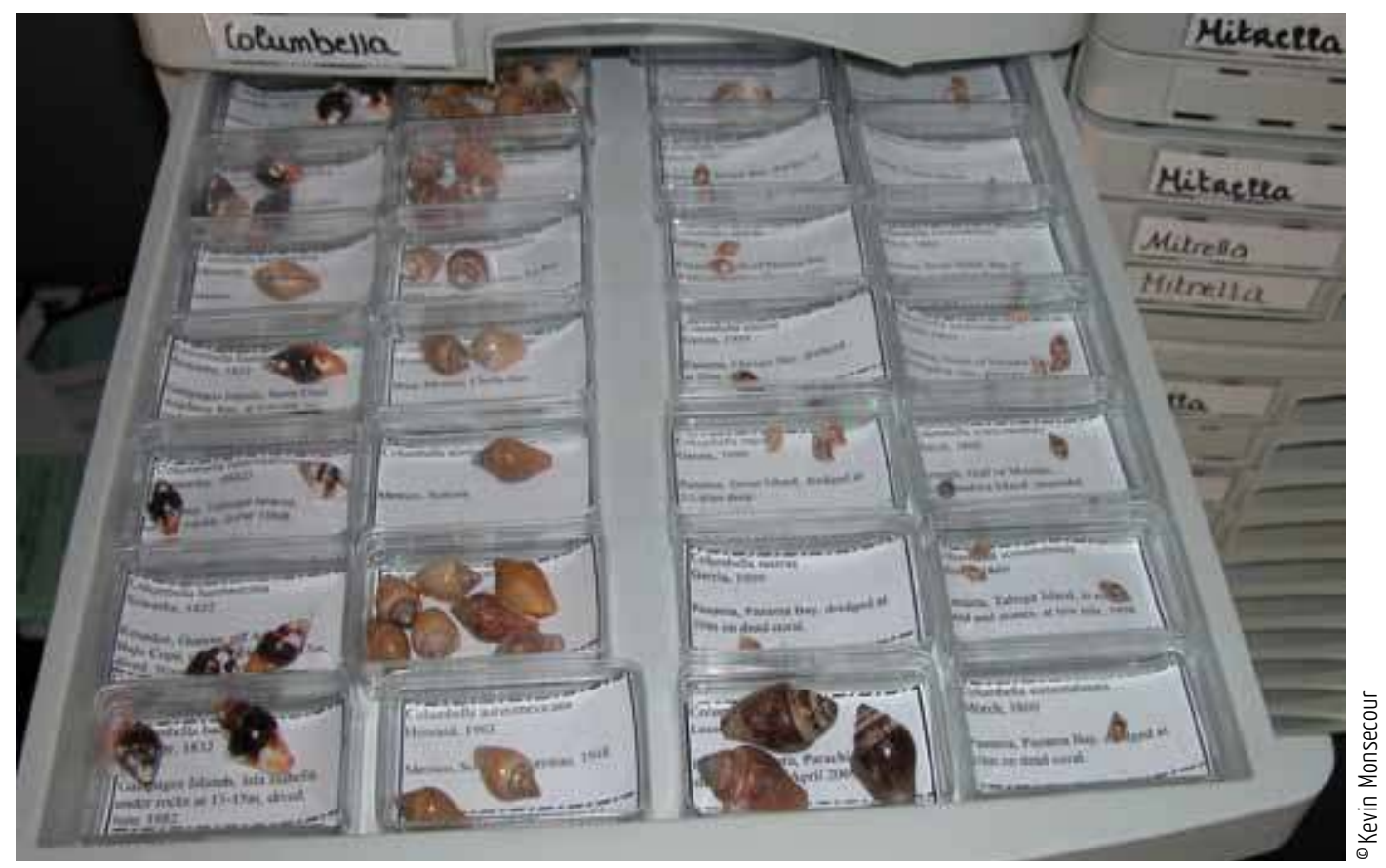

Sur la photo ci-dessus, un tiroir ouvert de la collection des Columbellidae de Kevin, à son domicile. Tous les tiroirs ressemblent à ces deux-là, avec au total, plus de 15000 spécimens dans sa collection personnelle, décembre 2012.
Très souvent leur curiosité naturaliste et leur goût de la collection ont été transmis par un proche, parent, ami, professeur qui a initié l'enfant ou l'adolescent à la géologie, à la paléontologie, à l'entomologie ou à la malacologie. Dès le départ, cet attrait naturaliste que certains qualifient de passion, s'inscrit dans des liens sociaux, dans des échanges, dans des sentiments et des affects vis-à-vis d'autres êtres humains qui jouent le rôle d'initiateur. Par amitié, par amour, par désir de plaire ou de faire plaisir à un proche, d'être admiré ou reconnu de lui, de devenir son ami, l'intérêt naturaliste se tisse et se consolide.

En 2000 son ami Koen l'a amené, avec son frère David, à la bourse aux coquillages de Paris et au Muséum pour rencontrer Philippe et son équipe. Sur les conseils de Guido, Kevin commençait alors à s'intéresser à un nouveau groupe, les Columbellidae, qui n’avait jusqu'alors aucun spécialiste. «Parce qu’elles étaient très jolies, pas chères et faciles à collectionner, j'ai commencé à m'intéresser aux Columbellidae » raconte Kevin.

Lorsque Philippe le rencontre, il est tout de suite intéressé par cette nouvelle spécialité. Et trois jours après avoir rencontré Kevin et David, Philippe leur envoie chez eux à Aarschot une boîte de cinquante centimètres carrés pleine de Columbellidae provenant des campagnes de Nouvelle-Calédonie, faites dans les années 1990.

En échange du travail de tri et d'identification qu'ils font sur ce matériel, Kevin sait qu'il pourra garder des paratypes pour sa collection personnelle. Il explique:

« Normalement quand il y a assez de paratypes pour le musée, on peut en garder un pour notre collection personnelle. Quand on a un exemplaire chez nous c'est plus facile pour comparer les spécimens et c'est pour ça que j’ai demandé à Philippe si je peux garder un spécimen de chaque espèce. [...] On travaille bénévolement pour le musée et ça nous coûte cher aussi parce que quand je viens au musée travailler sur les Columbellidae, c'est moi qui paye le TGV et l'hôtel. Mais c'est une passion d'étudier ces coquillages et ça donne aussi peut-être un peu de prestige. On peut dire oui j'ai 
décrit 60 espèces nouvelles! C'est vrai que l'on a des frais mais quand je vais avoir un exemplaire de chaque espèce, là ça devient très intéressant. Car certains spécimens coûtent 50 ou 100 euros pièce. Et du coup, à ce moment-là, on reçoit beaucoup d'argent du musée en fait! » (entretien avec Kevin, bourse aux coquillages de Paris, mars 2012).

Kevin semble ainsi dire que d'une certaine façon, à la fois symbolique mais aussi très matérielle, le Muséum le paye en monnaie... de coquillages. Car dans cet extrait d'entretien, même si ce n'est pas là sa motivation première, loin s'en faut, il sait que certains spécimens ont aussi une valeur marchande, et que du coup la valeur marchande de sa collection s'en accroît d'autant.

Ces dons de paratypes n'existent pas seulement entre professionnels et amateurs. Entre amateurs eux-mêmes, les coquillages circulent selon ce même chemin. Comme Kevin publie des descriptions de nouvelles espèces de Columbelles, beaucoup de collectionneurs et de marchands connaissent sa spécialité et lui envoient du matériel à identifier. Et, à chaque fois, en échange de ce travail d'identification, il peut garder des spécimens de chaque espèce.

« En Polynésie française, ils sont en train de faire un livre sur les coquillages et ils m’ont envoyé une quarantaine de lots de Columbelles. J'ai tout identifié et je peux garder un ou deux exemplaires de chaque espèce ce qui me fait 80 exemplaires pour moi. Du coup, c'est très intéressant de faire des manuscrits et d'écrire. Hier aussi j’ai reçu un sachet de Papouasie Nouvelle-Guinée et à mon avis, ce sont des espèces nouvelles. Je dois évidemment contrôler à la maison bien sûr, je ne décris pas sans contrôler. Mais tout ça c'est gratuit pour moi!

Le chemin des coquillages est ainsi le suivant : en décrivant une nouvelle espèce, Kevin choisit un spécimen type qu'il envoie au Muséum de Paris avec quelques paratypes. Il en conserve un ou deux pour sa collection personnelle. Et le marchand qui lui a envoyé ce matériel attend la publication de la description de l'espèce nouvelle pour commercialiser les spécimens qu'il a gardés. Comme on l'a déjà vu avec Luigi, la description d'une

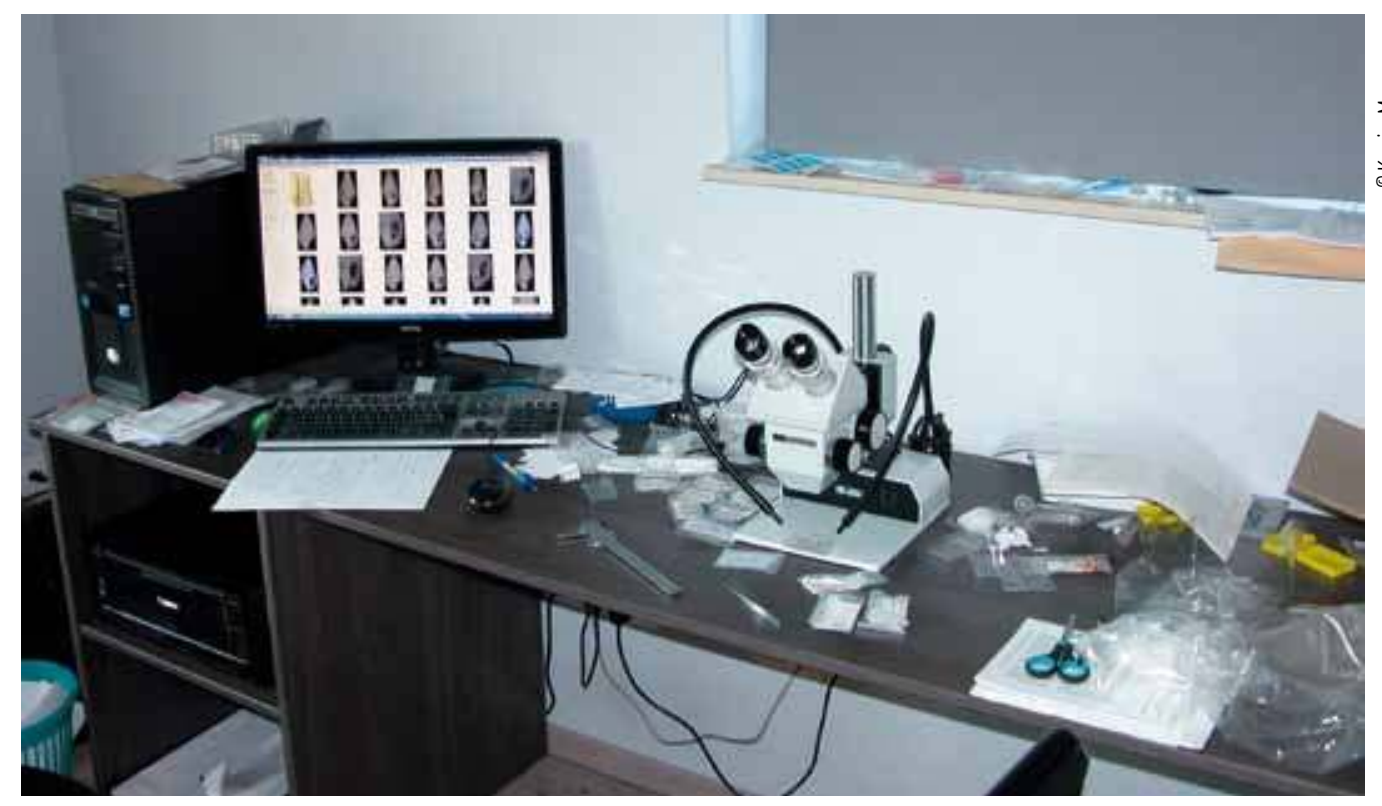

Le bureau de Kevin, chez lui, où il étudie les coquillages envoyés par le Muséum de Paris, et où il écrit ses articles. On peut voir l'habituel mélange de matériel hightech (loupe binoculaire, ordinateur) et lowtech (ciseaux, sachets en plastique). 


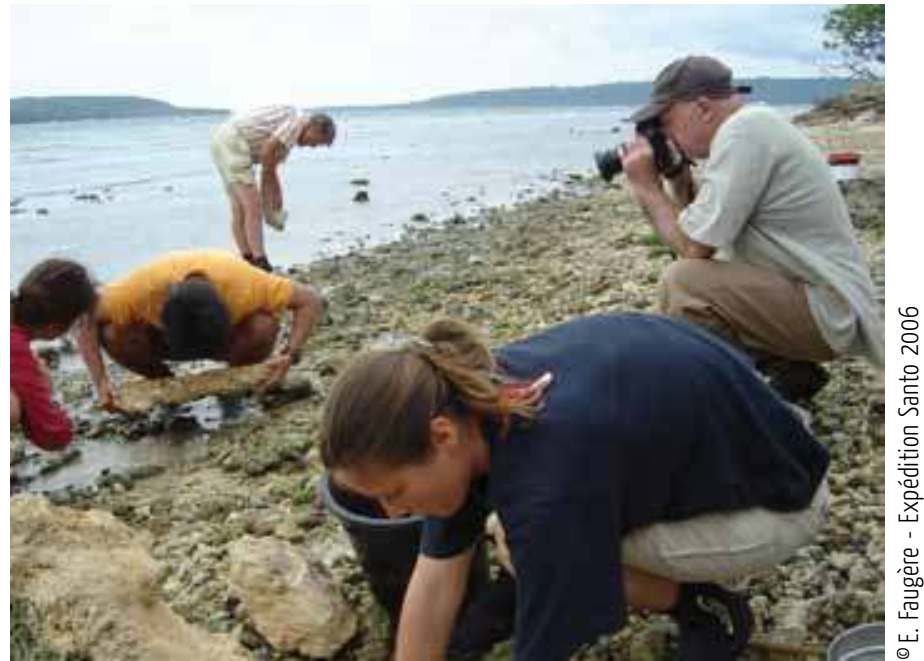

Une collecte à marée basse sur une plage de Luganville sur l'île d'Espiritu Santo lors de l'expédition Santo au Vanuatu dans le Pacifique Sud en octobre 2006. Au premier plan, Timéa, une doctorante allemande, soulève avec attention et concentration des pierres susceptibles de dissimuler les micros mollusques Acochlidia, sujets de sa thèse.

La veille, Takuma, l'étudiant japonais accroupi (en jaune) avait trouvé à cet endroit précis une nouvelle espèce d'Acochlidia, d'où la raison de leur présence au même endroit dès le lendemain, sous le regard attentif de Sarah, biologiste moléculaire au Muséum, et l'objectif d'un des photographes professionnels chargé de couvrir l'expédition.

Au fond, Anders, un malacologue suédois qui collabore de longue date à ces expéditions marines. espèce nouvelle lui donne une valeur marchande, même si, dans certains cas comme dans celui des Columbellidae cette valeur marchande est faible, de l'ordre de 50 euros maximum pour les espèces les plus rares. Par comparaison, dans les groupes les plus collectionnés où la demande est la plus forte, comme les porcelaines, les cônes ou les volutes, certains spécimens peuvent valoir plusieurs milliers d'euros.

Selon un autre amateur, Dominique, professeur de sport qui vit en Guadeloupe et qui est un grand collecteur et collectionneur:

« En général, quand j'ai plusieurs spécimens celui qui fait la description, je lui laisse le choix d'un spécimen. Il en prend un dans le lot. C'est un moyen pour eux de constituer leur collection. En général c'est ce qui se fait dans un lot quand il y a une vingtaine de pièces, celui qui va décrire l'espèce il en garde une ou deux pour lui. Ça lui permet je dirai en retour de se payer un peu d'une certaine manière et de garder une trace de son travail »

Ainsi dans cette circulation des coquillages, chacun, marchand, collectionneur-amateur de haut niveau, et professionnel malacologue tire son épingle du jeu et en sort gagnant.

\section{$\&$}

Cette circulation de coquillages, de types et de paratypes, les dons et les contre-dons qui se font entre professionnels et amateurs, mais aussi entre amateurs eux-mêmes, dessinent une économie morale spécifique à ces sciences naturalistes de terrain et de voyage. Si, dans cet article, et pour les besoins de ce Thema, je me suis centrée sur la seule circulation de coquillages, il existe bien d'autres types d'échanges au sein de cette communauté éclectique des malacologues qui participent aussi de cette économie morale. L'un d'entre eux consiste à donner à une espèce nouvelle de mollusque le nom d'un collecteur, d'un collectionneur, d'un mécène, d'un marchand, d'un amateur, d'un collègue (ou tout simplement d'un proche), en remerciement, en hommage, par amour, par amitié, etc. Le choix du nom d'une nouvelle espèce de mollusque dessine ainsi non seulement une cartographie de ses réseaux et liens professionnels et amicaux mais aussi de ceux de la communauté des malacologues: les plus connus d'entre eux sont généralement dotés de nombreux noms d'espèces voire, encore plus prestigieux, de noms de familles et de genres.

Ces circulations de coquillages, de noms, de services et d'échanges de toutes sortes construisent un collectif qui existe et perdure dans et par ces échanges tangibles, et notamment ces dons de coquillages. Au-delà de l'objet lui-même et de l'importance qu'il revêt aux yeux des malacologues amateurs et professionnels, ce qui semble compter autant si ce n'est davantage, ce sont les liens, d'amitié et de sociabilité, tissés autour et grâce à ces mollusques et à ces coquillages. Lors des nombreuses discussions que j'ai eues avec des amateurs-collectionneurs, parmi les principales raisons qu'ils donnaient au 
fait de venir chaque année à la bourse aux coquillages de Paris, parfois de très loin, c'était pour retrouver « les copains", partager et échanger entre amis autour de leur passion commune pour les mollusques et les coquillages. L'importance de cette sociabilité, masculine, est flagrante à la bourse de Paris où l'on voit des groupes de copains s'échanger des coquillages, se faire des dons, partager des analyses et des avis sur tel ou tel spécimen.

Si les liens se tissent aujourd'hui autour et à partir des coquillages, ils auraient aussi bien pu se tisser - ils l'ont parfois été dans le passé - autour d'autres objets comme les fossiles, les papillons, les minéraux, etc. Alors même qu'il occupe tant de place et d'importance dans la vie des malacologues amateurs et des professionnels, l'objet même de la collection, à savoir le coquillage semble parfois disparaître et se dissoudre pour ne laisser, in fine, que du lien. Qu'il s'agisse d'appartenir à une même «famille » comme me l'ont dit certains amateurs, ou à une même «maison » comme certains professionnels appellent le Muséum, les liens qu'ils ont tissé, depuis parfois des décennies, apparaissent particulièrement forts et solides, en partie parce qu'ils reposent aussi sur des voyages d'exploration et de collecte qui caractérisent les sciences de plein air et de terrain comme le sont les sciences naturalistes.
Deux amateurs de haut niveau : Mitsuo Chino, un collectionneur japonais, économiste dans une grande compagnie d'assurance du Japon, qui a commencé à s'intéresser aux coquillages à l'âge de 10 ans. Et Pierre Recourt, homme d'affaire hollandais, qui dirige une entreprise de fitness, et souhaite, après avoir vendu son entreprise, se consacrer à la malacologie et devenir un "amateur professionnel", « c'est-à-dire,

explique-t-il, quelqu'un qui a au moins le niveau d'un doctorat voire plus, et qui est le meilleur dans sa spécialité ». 


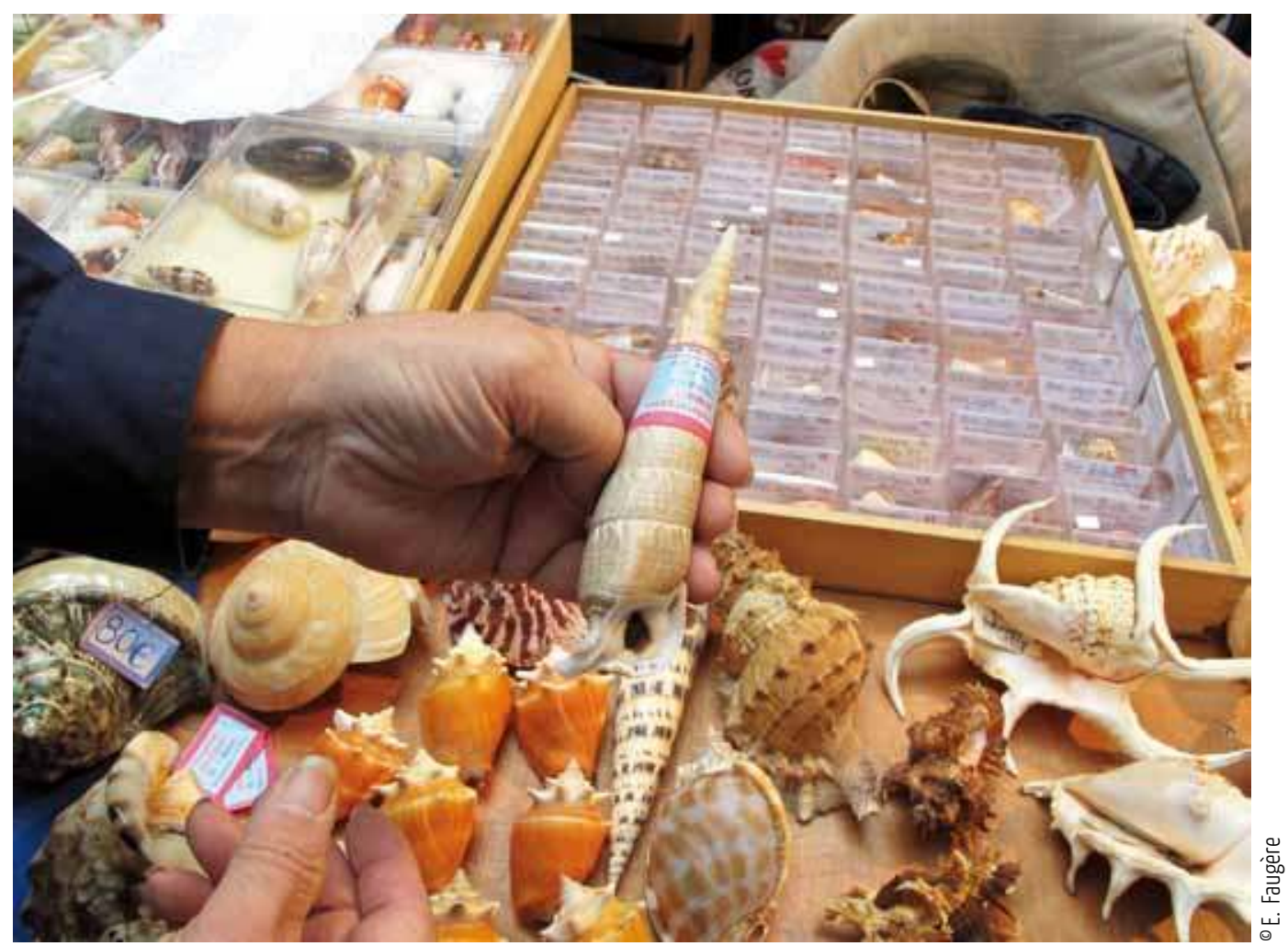

Rudo von Cosel, malacologue allemand, spécialiste des bivalves, proche collaborateur de Philippe Bouchet, regarde attentivement un Pseudovergatus nobilis, en provenance d'Indonésie, vendu 21 euros, à la bourse aux coquillages de Paris, mars 2011. 


\section{NOTES}

En ouverture: Un meuble à tiroirs de la collection des Columbellidae de Kevin Monsecour, à son domicile. Au total, plus de 15000 spécimens dans sa collection personnelle, décembre 2012.

1. En 2006 a eu lieu au Vanuatu la première grande expédition de ce nouveau genre, appelée l'expédition Santo 2006. De nombreuses publications sont issues de cette expédition, par exemple: Bouchet, Le Guyader, Pascal (eds.), 2011, Bouchet et al. (2008); Tardieu et Barnéoud 2007, Faugère 2008; Faugère et Louafi 2011, etc. Forts du succès scientifique et médiatique de l'expédition Santo 2006, les organisateurs ont lancé un programme décennal de grandes expéditions naturalistes dans les hotspots de la biodiversité des pays du sud, appelé « La Planète Revisitée ». Ce programme d'exploration est l'objet d'étude d'un projet de recherche (2010-2013) financé par l'Agence Nationale de la Recherche, programme « Sciences, Technologies et Savoirs en Sociétés. Enjeux actuels, questions historiques ». Ce projet de recherche que je coordonne, s'intitule « Expébiodiv. Étude pluridisciplinaire des grandes expéditions naturalistes contemporaines », ANR-09-SSOC-52. Cet article s'inscrit dans le cadre de ce projet.

2. La notion d'économie morale a été élaborée dans les années 1960 par un historien britannique Edward P. Thompson. Elle connaît depuis une quinzaine d'années un regain de vitalité notamment grâce aux travaux de Didier Fassin (cf. un article synthétique et récent paru en 2009 dans les Annales HSS). Dans le domaine des Social Studies of Science, c'est d'abord Lorraine Daston qui a utilisé la notion d'économie morale pour décrire les valeurs en vigueur dans l'univers de la science. Mais dans le domaine des Social Studies of Science, les travaux les plus intéressants me paraissent être ceux de Bruno Strasser.
3. Cet article repose sur l'étude du volet marin des grandes expéditions de La Planète Revisitée, au Vanuatu en 2006 et à Madagascar en 2010, ainsi que sur des enquêtes faites au laboratoire de malacologie du Muséum National d'Histoire Naturelle de Paris et à la Bourse aux Coquillages de Paris en 2011 et 2012. Le collectif dont il s'agit ici est celui qui lie les malacologues professionnels du MNHN à leur cercle d'amateurs.

4. L'holotype, appelé aussi « le type », est le spécimen à partir duquel une espèce a été décrite; les autres spécimens utilisés pour ce travail de description de l'espèce sont appelés « paratypes».

5. Dans les années 1980, l'ORSTOM (devenu IRD) et le Muséum National d'Histoire Naturelle de Paris ont lancé une série de campagnes océanographiques, appelées MUSORSTOM pour échantillonner les régions tropicales du Pacifique Sud et Ouest. Rebaptisées Tropical Deep Sea Benthos dans les années 2000, ces campagnes de collecte et d'exploration, uniques au monde, se poursuivent. Elles ont donné lieu à la publication d'une trentaine de volumes et la description de milliers d'espèces nouvelles; cf. notamment Bouchet, P., Héros, V., Lozouet, P., Maestrati, P., 2008, Un quart de siècle d'exploration des faunes malacologiques de profondeur dans le Pacifique Sud et Ouest: où en sommes-nous? Où allons-nous? in Xenophora, $n^{\circ} 126: 18-51$.

6. La Fondation Total mécène ses campagnes marines depuis l'an 2000 et la campagne Lifou en NouvelleCalédonie (cf. Faugère 2008).

\section{REMERCIEMENTS}

Je tiens à remercier ici chaleureusement Olivier Pascal et Philippe Bouchet pour la confiance qu'ils m'accordent depuis mars 2005, pour leur disponibilité, leur curiosité et leur ouverture d'esprit qui m’ont permis de participer aux expéditions terrestres et marines qu'ils organisent et d'avoir ainsi accès à un matériel empirique particulièrement riche. Pour les expéditions marines dont il est question dans cet article, je le dois aussi, bien évidemment, et je les en remercie ici vivement aux collaborateurs et collègues les plus anciens de Philippe Bouchet - Virginie Héros, Philippe Maestrati, Pierre Lozouet, Rudo von Cosel, Bertrand Richer de Forges -, comme les plus récents - Laure Corbari, Barbara Buge et Nicolas Puillandre notamment. Je remercie aussi tous les participants à ces expéditions, professionnels, amateurs, plongeurs, étudiants, ainsi que l'ensemble des bénévoles, des amateurs et des collectionneurs de coquillages qui ont toujours répondu avec beaucoup de bienveillance et de patience à toutes mes questions. Dans le cadre de cet article, je pense plus particulièrement à Luigi, Koen, Kevin, Denis, Bernard et Daniel, Dominique. Et je remercie enfin tous ceux qui ont accepté de figurer dans cet article ainsi que Reza et Thi Thao Amiraly, les parents de Sarah, et les propriétaires de l'Hôtel «Le Petit Bonheur» de Fort Dauphin à Madagascar où ont été accueillis et hébergés l'ensemble des participants du module marin en mai 2010. 


\section{RÉFÉRENCES}

Alliage 2011 « Amateurs». Alliage 69 [mis en ligne en octobre 2012] http://revel.unice.fr/alliage/index. html?id=3229 Consulté le 9 mars 2013.

Bory, A. 2008 De la générosité en entreprise. Mécénat et bénévolat des salariés dans les grandes entreprises en France et aux États-Unis, Thèse de sciences sociales, Paris 1.

Bouchet, P., Le Guyader, H., Pascal, O. (dir.) 2011 The Natural History of Santo, Collection Patrimoines Naturels. Paris : Muséum National d'Histoire Naturelle.

Bouchet, P., Héros, V., Lozouet, P. \& P. Maestrati 2008 Un quart de siècle d'exploration des faunes malacologiques de profondeur dans le Pacifique Sud et Ouest: où en sommes-nous? Où allons-nous? Xenophora 126.

Bouchet, P., Le Guyader, H., Pascal, O. 2008 Des voyages de Cook à l'expédition Santo 2006 : un renouveau des explorations naturalistes des îles du Pacifique. Journal de la Société des Océanistes 126-127.

Bouchet, P., Mermet, G. 2007 Regards sur les coquillages. Paris : Imprimerie Nationale Éditions : 97-98.

Charvolin, F. 2009 Comment penser les sciences naturalistes « à amateurs » à partir des passions cognitives. Natures, Sciences, Sociétés 17.

Charvolin, F., Micoud, A., Nyhart, L. (dir.) 2007 Des sciences citoyennes? La question de l'amateur dans les sciences naturalistes, La Tour d'Aigues, L'Aube.

Daston, L. 1995 The Moral Economy of Science. Osiris (10).

Fassin, D. 2009 Les économies morales revisitées. Annales HSS nº 6.

Faugère, E., Louafi, S. 2011 Le nouveau climat des expéditions naturalistes au Sud. L'exemple de Santo 2006 au Vanuatu. Revue Tiers Monde 207.

Faugère, E. 2008 L'exploration contemporaine de la biodiversité. Approche anthropologique de l'expédition Santo 2006. Journal de la Société des Océanistes 126-127.

Guilhot, N. 2004 Financiers, philanthropes. Vocations éthiques et reproduction du capital à Wall Street depuis 1970. Paris : Éditions Raison d'Agir.

Kohler, R. E. 1991 Partners in Science. Foundations and Natural Scientists 1900-1945. Chicago : The University of Chicago Press.

Kohler, R. E. 2006 All Creatures. Naturalists, Collectors, and Biodiversity, 1850-1950. Princeton and Oxford : Princeton University Press, .

Strasser, B. 2011 The Experimenter's Museum: GenBank, Natural History and the Moral Economies of Biomedicine. Isis (102) 1.

Strasser, B. 2012 Practices, styles and narratives: Collecting in the History of the Life Sciences. Osiris (à paraître).

Tardieu V., Barnéoud, L. 2007 Santo, les explorateurs de l'île planète. Paris : Belin. 


\section{RÉSUMÉ}

Échanges de coquillages entre amateurs et professionnels. L'économie morale des sciences naturalistes. Cet article repose sur l'étude du volet marin de grandes expéditions scientifiques et naturalistes organisées, depuis 2006, par le Muséum National d'Histoire Naturelle de Paris, l'ONG Pro-Natura International et l'Institut de Recherche pour le Développement. La présence de mécènes qui financent ces expéditions et d'amateurs bénévoles qui y participent m’a conduite à formuler l'hypothèse selon laquelle mécénat et bénévolat forment les deux piliers d'une économie morale spécifique à ces sciences naturalistes de terrain et de voyage. L'objectif de cet article, dans le cadre de ce Thema consacré aux itinéraires de coquillages, est ainsi de montrer que l'une des dimensions - peut-être même la principale - de cette économie morale réside dans des échanges et des dons de coquillages entre amateurs et professionnels de la malacologie, dont certains renouent avec l'ancienne fonction de monnaie de coquillages.

\section{ABSTRACT}

Shell exchanges between amateurs and professionals. The moral economy of naturalist sciences. This paper is based on the ethnographic study of the marine part of the great scientific and naturalists expeditions organized, since 2006, by the National Museum of Natural History of Paris, the NGO Pro-Natura International and the French "Institut de Recherche pour le Développement". Because these expeditions are sponsored by private foundations and many participants are amateurs as voluntary workers, I make the hypothesis that patronage and voluntary work are the two foundations of a specific moral economy of these naturalists' field sciences. The aim of this paper, in the framework of this Thema focused on the itineraries of the shells, is to show that one dimension - and probably the most important ones - of this moral economy lies in the exchanges and in the gifts and counter-gifts of shells between and among amateurs and professionals of the malacology, some of them reviving the old function of shells as money.

\section{MOTS CLÉS}

Coquillages, échanges, dons, économie morale, amateurs, professionnels, sciences naturalistes, expéditions

\section{KEYWORDS}

Shells, exchanges, gifts, moral economy, amateurs, professionals, naturalists sciences, expeditions 